Bull. Austral. Math. Soc.

26A45, 26A39, 26A42

Vol. $40(1989) \quad[147-155]$

\title{
GENERALISED HÖLDERIAN FUNCTIONS
}

\author{
S. De Sarkar and S. Panda
}

\begin{abstract}
The concept of $k$ th Hölderian functions on an interval $[a, b]$ which generalises the notion of Hölderian (Lipschitzian) functions of positive order on $[a, b]$ is introduced. The relationship of such functions to functions of bounded $k$ th variation and absolutely $k$ th continuous functions is examined. Properties induced by higher order derivatives in this new class of functions are investigated.
\end{abstract}

\section{INTRODUCTION}

It is well-known that a function $f$ of a real variable is said to be Hölderian (Lipschitzian) of order $\alpha>0$ on $[a, b]$ if there exists a positive constant $K$ such that for any two points $x$ and $y$ in $[a, b]$,

$$
|f(x)-f(y)| \leq K|x-y|^{\alpha} .
$$

If in this definition, $\alpha>1$, then $f$ is constant on $[a, b]$ and if $\alpha=1$ then $f$ is absolutely continuous (consequently of bounded variation) on $[a, b]$.

Russell [12] introduced the concept of functions of bounded $k$ th variation $\left(\mathrm{BV}_{k}\right.$ functions) on $[a, b]$ and studied their properties in detail. As a natural consequence the definition of absolutely $k$ th continuous functions $\left(\mathrm{AC}_{k}\right.$ functions) on $[a, b]$ was introduced by $D$ as and Lahiri [2] and they obtained sone interrelations between $B V_{k}$ and $\mathrm{AC}_{k}$ functions. The definitions of $\mathrm{BV}_{k}$ and $\mathrm{AC}_{k}$ functions involve higher order divided differences.

The main purpose of the present paper is to obtain a generalisation of Hölderian functions of positive order using higher order divided differences and to consider the development of some classical properties with such a framework. To do this, we first define $k$ th Hölderian functions of positive order on $[a, b]$. It is observed that such functions correspond to $\mathrm{BV}_{k}$ and $\mathrm{AC}_{k}$ functions in exactly the same way as Holderian functions of positive order correspond to functions of classical bounded variation and absolutely continuous functions. We then exhibit several results concerning higher order derivatives in this new class of functions.

For background and further information on $\mathrm{BV}_{k}$ and $\mathrm{AC}_{k}$ functions, we refer the reader to Russell $[13,14]$, De Sarkar and Das $[4,5,6,7]$, De Sarkar, Das and Lahiri [8] and Das and Das [3].

Received 28 October, 1988

Copyright Clearance Centre, Inc. Serial-fee code: 0004-9729/89 \$A2.00+0.00. 


\section{Preliminaries}

Let $f$ be a real-valued function defined on the closed interval $[a, b]$ and let $k$ be a positive integer greater than 1 . If $x_{0}, x_{1}, \ldots, x_{k}$ are any $(k+1)$ distinct points, not necessarily in linear order, in $[a, b]$, then the $k$ th divided difference of $f$ is defined by

$$
Q_{k}\left(f ; x_{0}, x_{1}, \ldots, x_{k}\right)=\sum_{i=0}^{k}\left[f\left(x_{i}\right) / \prod_{\substack{j=0 \\ j \neq i}}^{k}\left(x_{i}-x_{j}\right)\right]
$$

By a $\pi$-subdivision of $[a, b]$ we mean a finite set of points $x_{0}, x_{1}, \ldots, x_{n}$ in $[a, b]$ with $x_{0}<x_{1}<\ldots<x_{n}$ and we denote it by $\pi\left(x_{0}, x_{1}, \ldots, x_{n}\right)$. The number

$$
V_{k}(f ;[a, b])=\sup _{\pi} \sum_{i=0}^{n-k}\left(x_{i+k}-x_{i}\right)\left|Q_{k}\left(f ; x_{i}, \ldots, x_{i+k}\right)\right|
$$

where the supremum is taken for all $\pi$-subdivisions of $[a, b]$ is called the total kth variation of $f$ on $[a, b]$. If $V_{k}(f ;[a, b])<+\infty$, then $f$ is said to be of bounded kth variation, $\mathrm{BV}_{k}$, on $[a, b]$. For $x \in[a, b]$ we write $V_{k(f)}(x)=V_{k}(f ;[a, x])$.

Let $x_{1,0}<x_{1,1}<\ldots<x_{1, k} \leq x_{2,0}<x_{2,1}<\ldots<x_{2, k} \leq \ldots \leq x_{n, 0}<x_{n, 1}<$ $\ldots<x_{n, k}$ be any subdivision of $[a, b]$ where $x_{i, j} \in[a, b]$. We say that the intervals $\left(x_{i, 0}, x_{i, k}\right), i=1,2, \ldots, n$ form an elementary system $I$, say, in $[a, b]$. The system is denoted by

$$
I\left(x_{i, 1}, \ldots, x_{i, k-1}\right):\left(x_{i, 0}, x_{i, k}\right), \quad i=1,2, \ldots, n
$$

We write

$$
\sigma|I|=\sum_{i=1}^{n}\left(x_{i, k}-x_{i, 0}\right)\left|Q_{k}\left(f ; x_{i, 0}, \ldots, x_{i, k}\right)\right|
$$

and

$$
m I=\sum_{i=1}^{n}\left(x_{i, k}-x_{i, 0}\right)
$$

The function $f$ is said to be absolutely kth continuous, $\mathrm{AC}_{k}$, on $[a, b]$ if for $\varepsilon>0$, arbitrary, there exists $\delta(\varepsilon)>0$ such that for any elementary system $I$ in $[a, b]$ with $m I<\delta$ the relation $\sigma|I|<\varepsilon$ is satisfied.

Let $x_{0}$ be any point in $[a, b]$ and let $x_{1}, x_{2}, \ldots, x_{k}$ be any set of $k$ distinct points in $[a, b]$ with the property $0<\left|x_{1}-x_{0}\right|<\left|x_{2}-x_{0}\right|<\ldots<\left|x_{k}-x_{0}\right|$. If the iterated limit

$$
\lim _{x_{k} \rightarrow x_{0}} \ldots \lim _{x_{1} \rightarrow x_{0}} k ! Q_{k}\left(f ; \dot{x}_{0}, x_{1}, \ldots, x_{k}\right)
$$

exists, then this limit is called the kth Reimann ${ }^{\star}$ derivative of $f$ at $x_{0}$ and is denoted by $D^{k} f\left(x_{0}\right)$. The ordinary derivative of $f$ of order $r$ at $x_{0}$ is denoted by $f^{r}\left(x_{0}\right)$. We note that when $f^{r}\left(x_{0}\right)$ exists, then $D^{r} f\left(x_{0}\right)$ exists, and $f^{r}\left(x_{0}\right)=D^{r} f\left(x_{0}\right)$. 


\section{3. $k$ TH HöldERIAN FUNCTIONS}

Definition 3.1: The function $f$ is said to be kth.Holderian of order $\alpha>0$ with the constant $M>0$ on $[a, b]$ if for any set of $(k+1)$ points $x_{0}<x_{1}<\ldots<x_{k}$ in $[a, b]$ we have

$$
\left|Q_{k-1}\left(f ; x_{0}, x_{1}, \ldots, x_{k-1}\right)-Q_{k-1}\left(f ; x_{1}, x_{2}, \ldots, \dot{x}_{k}\right)\right| \leq M\left(x_{k}-x_{0}\right)^{\alpha} .
$$

In this case we write $f \in H_{k}(\alpha, M,[a, b])$.

We note that for $k=1$, the above definition gives the definition of Hölderian functions of order $\alpha$ on $[a, b]$. When $\alpha>1$, by using Lemma 4 of Russell [12], it is easy to show that $D^{k} f(x)=0$ for all $x \in[a, b]$ and consequently $f$ is a polynomial of degree $(k-1)$ at most.

Theorem 3.1. If $f \in H_{k}(\alpha, M,[a, b])$, with $\alpha>0$, then $f^{k-1}$ exists and is continuous in $[a, b]$.

Proof: Let $a<c<b$ and $\varepsilon>0$ be arbitrary. We choose points

$$
x_{p-k+1}<x_{p-k+2}<\ldots<x_{p} \neq c<x_{p+1}<\ldots<x_{p+k+1}
$$

such that

$$
\left(x_{p+k-1}-x_{p-k+1}\right)<(\varepsilon /(k-1) ! M(k-1))^{1 / \alpha} .
$$

Then we have

$\left|Q_{k-1}\left(f ; x_{i}, \ldots, x_{i+k-1}\right)-Q_{k-1}\left(f ; x_{i+1}, \ldots, x_{i+k}\right)\right| \leq M\left(x_{i+k}-x_{i}\right)^{\alpha}<\varepsilon /(k-1) !(k-1)$

for $i=p-k+1, \ldots, p-1$. Now combining $(k-1)$ inequalities we obtain

$$
\left|(k-1) ! Q_{k-1}\left(f ; x_{i}, \ldots, x_{i+k-1}\right)-(k-1) ! Q_{k-1}\left(f ; x_{j}, \ldots, x_{j+k-1}\right)\right|<\epsilon
$$

for $i=p-k+1, \ldots, p$ and $j=p-k+1, \ldots, p$. Therefore, $D^{k-1} f(c)$ exists and hence $D^{k-1} f(x)$ exists in $(a, b)$. By suitable modifications it can be shown that at the end points $a$ and $b, D^{k-1} f$ (of course one-sided) exists. It is easy to see that there exists a $\delta$ with $0<\delta<(\varepsilon / k ! M)^{1 / a}$ such that for $c, d \in[a, b]$,

$$
\left|D^{k-1} f(c)-D^{k-1} f(d)\right|<\varepsilon
$$

whenever $|c-d|<\delta$ and so $D^{k-1} f$ is continuous in $[a, b]$. The theorem now follows from Theorem 4.4 of Bullen and Mukhopadhyay [1] and Theorem 3 of Oliver [11].

The following result is immediate. 
TheOREM 3.2. If $f \in H_{k}(1, M,[a, b])$, then $f$ is $A C_{k}$ on $[a, b]$.

THEOREM 3.3. If the $k$ th divided differences of $f$ are bounded on $[a, b]$ then $f \in H_{\boldsymbol{k}}(1, M,[a, b])$.

Proof: The proof follows readily from Lemma 4 of Russell [12].

In connection with Theorem 4 of Russell [12] and Theorem 3.3 above we observe that if $f$ is $\mathrm{BV}_{k}$ on $[a, b]$, then $f$ may not be $k$ th Hölderian of order 1 with any positive constant $M$ on $[a, b]$. Functions which are $\mathrm{BV}_{k}$ but not $\mathrm{AC}_{k}$ (see Example 2.2 of $\mathrm{De}$ Sarkar and Das [4]) are examples of such functions.

THeonem 3.4. If $f \in H_{k}(1, M,[a, b])$, then $V_{k(f)} \in H_{1}(1, k M,[a, b])$.

Proof: Let $x_{1}$ and $x_{2}$ be any two points in $[a, b]$ with $x_{1}<x_{2}$. Consider any $\pi\left(z_{0}, z_{1}, \ldots, z_{n}\right)$ subdivision of $\left[x_{1}, x_{2}\right]$. We then have $k$ sets of non-overlapping intervals $\left(z_{i}, z_{i+k}\right), i \in T_{r}=\{r, k+r, 2 k+r, \ldots \leq n\}$ and $r=0,1, \ldots, k-1$ so that

$$
\begin{aligned}
& \sum_{i=0}^{n-k}\left|Q_{k-1}\left(f ; z_{i}, \ldots, z_{i+k-1}\right)-Q_{k-1}\left(f ; z_{i+1}, \ldots, z_{i+k}\right)\right| \\
& \quad=\sum_{r=0}^{k-1} \sum_{i \in T_{r}}\left|Q_{k-1}\left(f ; z_{i}, \ldots, z_{i+k-1}\right)-Q_{k-1}\left(f ; z_{i+1}, \ldots, z_{i+k}\right)\right| .
\end{aligned}
$$

Since $f \in H_{k}(1, M,[a, b])$ it follows that

$$
\begin{aligned}
& \sum_{i=0}^{n-k}\left|Q_{k-1}\left(f ; z_{i}, \ldots, z_{i+k-1}\right)-Q_{k-1}\left(f ; z_{i+1}, \ldots, z_{i+k}\right)\right| \\
& \quad \leq M \sum_{r=0}^{k-1} \sum_{i \in T_{r}}\left(z_{i+k}-z_{i}\right) \\
& \quad \leq k M\left(x_{2}-x_{1}\right) .
\end{aligned}
$$

Therefore $V_{k}\left(f ;\left[x_{1}, x_{2}\right]\right) \leq k M\left(x_{2}-x_{1}\right)$. This implies, in view of Theorem 7 of Russell [12] that

$$
\left|V_{k(f)}\left(x_{2}\right)-V_{k(f)}\left(x_{1}\right)\right| \leq k M\left(x_{2}-x_{1}\right)
$$

and thus the theorem is proved.

TheOREM 3.5. If $V_{k(f)} \in H_{1}(\alpha, M,[a, b])$ with $\alpha>0$, then $f \in H_{k}(\alpha, M,[a, b])$.

ProOf: For any set of $(k+1)$ points $y_{0}<y_{1}<\ldots<y_{k}$ in $[a, b]$ we have, using Theorem 5 of Russell [12],

$$
\begin{aligned}
\mid Q_{k-1} & \left(f ; y_{0}, y_{1}, \ldots, y_{k-1}\right)-Q_{k-1}\left(f ; y_{1}, y_{2}, \ldots, y_{k}\right) \mid \\
& \leq V_{k}\left(f ;\left[y_{0}, y_{k}\right]\right) \\
& \leq\left|V_{k(f)}\left(y_{k}\right)-V_{k(f)}\left(y_{0}\right)\right|
\end{aligned}
$$


and so the theorem is proved.

THEOREM 3.6. If $f \in H_{k}(\alpha, M,[a, b]), \alpha>0$, then $f^{1} \in H_{k-1}(\alpha,(3 k-5) M,[a, b])$.

Proof: By Theorem 3.1, $f^{k-1}$ exists on $[a, b]$. For any set of $k$ points $x_{0}<x_{1}<$ $\ldots<x_{k-1}$ in $[a, b]$ we have, using Theorem 8 of Russell [12],

$$
\begin{gathered}
\left|Q_{k-2}\left(f^{1} ; x_{0}, x_{1}, \ldots, x_{k-2}\right)-Q_{k-2}\left(f^{1} ; x_{1}, x_{2}, \ldots, x_{k-1}\right)\right| \\
=\left|\sum_{k=0}^{k-2} Q_{k-1}\left(f ; x_{0}, \ldots, x_{h}, x_{h}, \ldots, x_{k-2}\right)-\sum_{h=1}^{k-1} Q_{k-1}\left(f ; x_{1}, \ldots, x_{h}, x_{h}, \ldots, x_{k-1}\right)\right| \\
=\left|\sum_{t=0}^{k-2}\left\{Q_{k-1}\left(f ; x_{0}, \ldots, x_{t}, x_{t}, \ldots, x_{k-2}\right)-Q_{k-1}\left(f ; x_{1}, \ldots, x_{t+1}, x_{t+1}, \ldots, x_{k-1}\right)\right\}\right| \\
\leq \sum_{t=0}^{k-2}\left|Q_{k-1}\left(f ; x_{0}, \ldots, x_{t}, x_{t}, \ldots, x_{k-2}\right)-Q_{k-1}\left(f ; x_{1}, \ldots, x_{t+1}, x_{t+1}, \ldots, x_{k-1}\right)\right| \\
\leq \sum_{t=0}^{k-2}\left\{\left|Q_{k-1}\left(f ; x_{0}, \ldots, x_{t}, x_{t}, \ldots, x_{k-2}\right)-Q_{k-1}\left(f ; x_{0}, \ldots, x_{t}, \xi_{t+1}, \ldots, x_{k-2}\right)\right|\right. \\
\quad+\left|Q_{k-1}\left(f ; x_{1}, \ldots, x_{t+1}, x_{t+1}, \ldots, x_{k-1}\right)-Q_{k-1}\left(f ; x_{1}, \ldots, x_{t+1}, \xi_{t+1}, \ldots, x_{k-1}\right)\right| \\
\left.\quad+\left|Q_{k-1}\left(f ; x_{0}, \ldots, x_{t}, \xi_{t}, \ldots, x_{k-2}\right)-Q_{k-1}\left(f ; x_{1}, \ldots, x_{t+1}, \xi_{t+1}, \ldots, x_{k-1}\right)\right|\right\}
\end{gathered}
$$

where $x_{s}<\xi_{s}<x_{s+1}$ when $s=0,1, \ldots, k-2$ and $x_{s-1}<\xi_{s}<x_{s}$ when $s=k-1$. For $\varepsilon>0$, arbritary, the points $\xi_{0}<\xi_{1}<\ldots<\xi_{k-1}$ can be chosen so that

$$
\left|Q_{k-1}\left(f ; x_{0}, \ldots, x_{t}, x_{t}, \ldots, x_{k-2}\right)-Q_{k-1}\left(f ; x_{0}, \ldots, x_{t}, \xi_{t}, \ldots, x_{k-2}\right)\right|<\varepsilon / 2(k-1)
$$

and

$$
\begin{aligned}
& \left|Q_{k-1}\left(f ; x_{1}, \ldots, x_{t+1}, x_{t+1}, \ldots, x_{k-1}\right)-Q_{k-1}\left(f ; x_{1}, \ldots, x_{t+1}, \xi_{t+1}, \ldots, x_{k-1}\right)\right| \\
& \quad<\varepsilon / 2(k-1)
\end{aligned}
$$

when $t=0,1, \ldots, k-2$. Thus we get

(3.1) $\left|Q_{k-2}\left(f^{1} ; x_{0}, x_{1}, \ldots, x_{k-2}\right)-Q_{k-2}\left(f^{1} ; x_{1}, x_{2}, \ldots, x_{k-1}\right)\right|$

$$
<\sum_{t=0}^{k-2}\left|Q_{k-1}\left(f ; x_{0}, \ldots, x_{t}, \xi_{t}, \ldots, x_{k-2}\right)-Q_{k-1}\left(f ; x_{1}, \ldots, x_{t+1}, \xi_{t+1}, \ldots, x_{k-1}\right)\right|+\varepsilon
$$

Consider

$$
\left|Q_{k-1}\left(f ; x_{0}, \ldots, x_{t}, \xi_{t}, \ldots, x_{k-2}\right)-Q_{k-1}\left(f ; x_{1}, \ldots, x_{t+1}, \xi_{t+1}, \ldots, x_{k-1}\right)\right|
$$


for a fixed value of $t$ where $0 \leqslant t \leqslant k-2$. When $t=0$ we have

$$
\begin{aligned}
\left|Q_{k-1}\left(f ; x_{0}, \ldots, x_{t}, \xi_{t}, \ldots, x_{k-2}\right)-Q_{k-1}\left(f ; x_{1}, \ldots, x_{t+1}, \xi_{t+1}, \ldots, x_{k-1}\right)\right| \\
=\left|Q_{k-1}\left(f ; x_{0}, \xi_{0}, x_{1}, \ldots, x_{k-2}\right)-Q_{k-1}\left(f ; x_{1}, \xi_{1}, x_{2}, \ldots, x_{k-1}\right)\right|
\end{aligned}
$$

We take $y_{0}=x_{0}, y_{1}=\xi_{0}, y_{2}=x_{1}, y_{3}=\xi_{1}, y_{r}=x_{r-2} ; r=4,5, \ldots,(k+1)$. Then, clearly, $y_{0}<y_{1}<\ldots<y_{k+1}$. By Theorem 1 of Russell [12] there exists a number $\beta$ with $0<\beta<1$ such that

$$
\begin{aligned}
\mid Q_{k-1}( & \left.f ; x_{0}, \xi_{0}, x_{1}, \ldots, x_{k-2}\right)-Q_{k-1}\left(f ; x_{1}, \xi_{1}, x_{2}, \ldots, x_{k-1}\right) \mid \\
= & \left|Q_{k-1}\left(f ; y_{0}, y_{1}, y_{2}, y_{4}, y_{5}, \ldots, y_{k}\right)-Q_{k-1}\left(f ; y_{2}, y_{3}, \ldots, y_{k+1}\right)\right| \\
= & \mid \beta Q_{k-1}\left(f ; y_{0}, y_{1}, y_{2}, y_{3}, \ldots, y_{k-1}\right)+(1-\beta) Q_{k-1}\left(f ; y_{1}, y_{2}, \ldots, y_{k}\right) \\
& \quad-Q_{k-1}\left(f ; y_{2}, y_{3}, \ldots, y_{k+1}\right) \mid \\
\leq & \left|Q_{k-1}\left(f ; y_{0}, y_{1}, \ldots, y_{k-1}\right)-Q_{k-1}\left(f ; y_{1}, y_{2}, \ldots, y_{k}\right)\right| \\
& \quad+\left|Q_{k-1}\left(f ; y_{1}, y_{2}, \ldots, y_{k}\right)-Q_{k-1}\left(f ; y_{2}, y_{3}, \ldots, y_{k+1}\right)\right| \\
\leq & M\left(y_{k}-y_{0}\right)^{\alpha}+M\left(y_{k+1}-y_{1}\right)^{\alpha} \\
< & 2 M\left(y_{k+1}-y_{0}\right)^{\alpha}=2 M\left(x_{k-1}-x_{0}\right)^{\alpha} .
\end{aligned}
$$

When $1 \leqslant t \leqslant k-3$ we define

$$
\begin{aligned}
y_{j} & =x_{j} ; \quad j=0,1, \ldots, t \\
y_{t+1} & =\xi_{t}, \quad y_{t+2}=x_{t+1}, \quad y_{t+3}=\xi_{t+1}, \\
y_{t+r} & =x_{t+r-2} ; \quad r=4,5, \ldots,(k+1-t) .
\end{aligned}
$$

With this relabelling, using Theorem 1 of Russell [12], it can be shown in a similar manner that for $1 \leqslant t \leqslant k-3$,

$$
\begin{aligned}
& \left|Q_{k-1}\left(f ; x_{0}, \ldots, x_{t}, \xi_{t}, \ldots, x_{k-2}\right)-Q_{k-1}\left(f ; x_{1}, \ldots, x_{t+1}, \xi_{t+1}, \ldots, x_{k-1}\right)\right| \\
& \quad<3 M\left(x_{k-1}-x_{0}\right)^{\alpha} .
\end{aligned}
$$

For $t=k-2$ we consider

$$
\left|Q_{k-1}\left(f ; x_{0}, x_{1}, \ldots, x_{k-2}, \xi_{k-2}\right)-Q_{k-1}\left(f ; x_{1}, x_{2}, \ldots, x_{k-1}, \xi_{k-1}\right)\right|
$$

Since $x_{k-2}<\xi_{k-1}<x_{k-1}$, in view of Lenma 3 of Russell [12], we have

$$
\begin{aligned}
& \left|Q_{k-1}\left(f ; x_{0}, \dot{x}_{1}, \ldots, x_{k-2}, \xi_{x-2}\right)-Q_{k-1}\left(f ; x_{1}, x_{2}, \ldots ; x_{k-1}, \xi_{k-1}\right)\right| \\
& \quad=\left|Q_{k-1}\left(f ; x_{0}, x_{1}, \ldots, x_{k-2}, \xi_{k-2}\right)-Q_{k-1}\left(f ; x_{1}, x_{2}, \ldots, x_{k-2}, \xi_{k-1}, x_{k-1}\right)\right| .
\end{aligned}
$$


Relabelling the set of points as

$$
\begin{aligned}
x_{j} & =y_{j} ; \quad j=0,1, \ldots, k-2, \\
y_{k-1} & =\xi_{k-2}, \quad y_{k}=\xi_{k-1}, \quad y_{k+1}=x_{k-1}
\end{aligned}
$$

and using Theorem 1 of Russell [12] again, we can show that

$$
\begin{aligned}
& \left|Q_{k-1}\left(f ; x_{0}, x_{1}, \ldots, x_{k-2}, \xi_{k-2}\right)-Q_{k-1}\left(f ; x_{1}, x_{2}, \ldots, x_{k-1}, \xi_{k-1}\right)\right| \\
& \quad<2 M\left(x_{k-1}-x_{0}\right)^{\alpha} .
\end{aligned}
$$

Hence from (3.1) we obtain

$$
\begin{aligned}
& \mid Q_{k-1}\left(f^{1} ; x_{0}, x_{1}, \ldots, x_{k-2}\right)-Q_{k-1}\left(f^{1} ; x_{1}, x_{2}, \ldots, x_{k-1}\right) \\
& \quad<(3 k-5) M\left(x_{k-1}-x_{0}\right)^{\alpha}+\varepsilon
\end{aligned}
$$

As $\varepsilon>0$ is arbitrary, the theorem is proved.

The following corollary. follows by straightforward induction.

Corollany 3.1. If $f \in H_{k}(\alpha, M,[a, b])$ with $\alpha>0$, then $f^{r} \in H_{k-r}\left(\alpha, M_{r},[a, b]\right)$ where $M_{r}=(3 k-5)(3 k-8) \ldots(3 k-3 r-2) M$ for $r=$ $1,2, \ldots,(k-1)$.

ThEOREM 3.7. If $f^{k-1} \in H_{1}(\alpha, M,[a, b])$ with $\alpha>0$, then $f \in H_{k}(\alpha, M /(k-1) !,[a, b])$.

Proof: Let $x_{0}<x_{1}<\ldots<x_{k}$ be any set of $(k+1)$ points in $[a, b]$. By MilneThomson $[10, p .6], f^{k-1}$ possesses the mean value property on $[a, b]$, namely, for any set of $k$ points $y_{1}<y_{2}<\ldots<y_{k}$ in $[a, b]$ there exists at least one $\eta$ in $\left(y_{1}, y_{k}\right)$ such that

$$
(k-1) ! Q_{k-1}\left(f ; y_{1}, y_{2}, \ldots, y_{k}\right)=f^{k-1}(\eta) .
$$

Since $f^{k-1} \in H_{1}(\alpha, M[a, b])$ we thus have

$$
\begin{aligned}
\mid Q_{k-1} & \left(f ; x_{0}, x_{1}, \ldots, x_{k-1}\right)-Q_{k-1}\left(f ; x_{1}, x_{2}, \ldots, x_{k}\right) \mid \\
& =[1 /(k-1) !]\left|f^{k-1}\left(\eta_{1}\right)-f^{k-1}\left(\eta_{2}\right)\right| ; \quad \eta_{1} \in\left(x_{0}, x_{k-1}\right), \quad \eta_{2} \in\left(x_{1}, x_{k}\right) \\
& \leq[M /(k-1) !]\left|\eta_{1}-\eta_{2}\right|^{\alpha} \\
& \leq[M /(k-1) !]\left(x_{k}-x_{0}\right)^{\alpha}
\end{aligned}
$$

and this completes the proof.

Using Theorem 3.6 we get the following corollary. 
Corollaky 3.2. If $f^{r} \in H_{k-r}(\alpha, N,[a, b])$ with $\alpha>0$, for some $r$ such that $1 \leq$ $r \leq k-2$, then $f \in H_{k}\left(\alpha, N_{r},[a, b]\right)$, where $N_{r}=[1.4 \ldots(3 k-3 r-5) N] /[(k-1) !]$.

For $0<\alpha<1, f \in H_{k}(\alpha, M,[a, b])$ does not imply that $f$ is $\mathrm{BV}_{k}$ on $[a, b]$. To see this, consider the following example.

Example 3.1. Let $0<\alpha<1$. Let $f$ be the function on $[0,1]$ such that $f^{1}=F$ where $F^{\prime}$ is the Weierstrass non-differentiable function defined by

$$
F(x)=\sum_{n=0}^{\infty} p^{-n} \sin 2 \pi q^{n} x
$$

where $p>1, q$ is even and $q>(4 \pi+1) p$, with $p=q^{\alpha}$. Taking

$$
M=[(2 \pi p q) / \dot{(} q-p)]+[(2 p) /(p-1)]
$$

we see (Hardy [9]) that $f^{1} \in H_{1}(\alpha, M,[0,1])$. Thus, by Theorem 3.7, $f \in H_{2}(\alpha, M,[0,1])$.

Since $f^{1}$ is not BV on $[0,1]$, it follows from Theorem 7 of Russell [14] that $f$ is not $\mathrm{BV}_{2}$ on $[0,1]$.

We find the following theorem useful.

THEOREM 3.8. If $f^{k-1}$ is absolutely continuous (AC) on $[a, b]$, then $f$ is $A C_{k}$ on $[a, b]$.

Proof: Consider an elementary system $I\left(x_{i, 1}, \ldots, x_{i, k-1}\right):\left(x_{i, 0}, x_{i, k}\right), i=1,2$, $\ldots, n$ in $[a, b]$. Then in view of Lemma 4 of Russell $[12]$ we have

$$
\sigma|I|=\sum_{i=1}^{n}\left|Q_{k-1}\left(f ; x_{i, 1}, \ldots, x_{i, k}\right)-Q_{k-1}\left(f ; x_{i, 0}, \ldots, x_{i, k-1}\right)\right| .
$$

Using Milne-Thomson $[10, p .6]$ it is then easy to see that

$$
\sigma|I|=[1 /(k-1) !]\left|f^{k-1}\left(\beta_{i}\right)-f^{k-1}\left(\gamma_{i}\right)\right|
$$

where $\left(\gamma_{i}, \beta_{i}\right)$, for $i=1,2, \ldots, n$ is a sequence of non-overlapping intervals in $[a, b]$. The rest of the proof is straightforward.

We conclude with the following example, which shows that the converse of Theorem 3.2 is, in general, not true.

Example 3.2. Consider the function

$$
f(x)=\left[2^{k-1} \sqrt{x^{2 k-1}}\right] /[3.5 \ldots(2 k-1)]
$$

on $[0,1]$. Then $f^{k-1}(x)=\sqrt{x}$ is absolutely continuous $(A C)$ on $[0,1]$ and so, by Theorem 3.8, $f$ is $\mathrm{AC}_{k}$ on $[0,1]$. For $0<x \leq 1$ we have

$$
\left.\left\|f^{k-1}(x)-f^{k-1}(0)\right\|\right] /[x-0]=1 / \sqrt{x},
$$

which is unbounded as $x \rightarrow 0$. Consequently $f^{k-1} \notin H_{1}(1, M,[0,1])$ for any $M>0$ and so, by Theorem 3.5, $f \notin H_{k}(1, M,[0,1])$ for any $M>0$. 


\section{REFERENCES}

[1] P.S. Bullen and S.N. Mukhopadhya, 'Relations between some general $n$th order derivatives', Fund. Math. 80 (1974), 257-276.

[2] A.G. Das and B.K. Lahiri, 'On absolutely $k$ th continuous functions', Fund. Math. 105 (1980), 159-169.

[3] U. Das and A.G. Das, 'Convergence in kth variation and $R S_{k}$ integrals', J. Austral. Math. Soc. Ser. $A 31$ (1981), 163-174.

[4] S. De Sarkar and A.G. Das, 'On functions of bounded $k$ th variation', $J$. Indian Inst. Sci. 64(B) (1983), 299-309.

[5] S. De Sarkar and A.G. Das, 'A convergence theorem in essential $k$ th variation', Soochow J. Math. 11 (1985), 51-57.

[6] S. De Sarkar and A.G. Das, 'On functions of bounded essential $k$ th variation', Bull. Calcutta Math. Soc. 78 (1986), 249-258.

[7] S. De Sarkar and A.G. Das, 'Riemann derivatives and general integrals', Bull. Austral. Math. Soc. 36 (1987), 187-211.

[8] S. De Sarkar, A.G. Das and B.K. Lahiri, 'Approximate Riemann" derivative and approximate $P^{k}-, D^{k}$ - integrals', Indian J. Math. 27 (1985), 1-32.

[9] G.H. Hardy, 'Weierstrass's non-differentiable function', Trans. Amer. Math. Soc. 17 (1916), 301-325.

[10] L.M. Milne-'Thomson, The calculus of finite differences (Chelsea, New York, 1981 ).

[11] H.V. Oliver, 'The exact Peano derivative', Trans. Amer. Math. Soc. 76 (1954), 444-456.

[12] A.M. Russell, 'Functions of bounded kth variation', Proc. London Math. Soc. (3)26 (1973), 547-563.

[13] A.M. Russell, 'An integral representation for a generalised variation of a function', Bull. Austral. Math. Soc. 11 (1974), 225-229.

[14] A.M. Russell, 'Further results on an integral representation of functions of generalised variation', Bull. Austral. Math. Soc. 18 (1978), 407-420.

Dr. S. De Sarkar,

Department of Mathematics, University of Kalyani,

Kalyani - 741 235,

Dist. Nadia, West Bengal,

India 